The Egyptian Journal of Hospital Medicine (October 2020) Vol. 81 (3), Page 1628-1633

\title{
Evaluation of the Association between Acne Vulgaris and Metabolic Syndrome in Adolescents at Zagazig University Hospitals
}

Ghada Ibrahim Ahmed, Ayman Elsayed Yousef, Eman Salah El-Din

Department of Dermatology, Venereology \& Andrology, Faculty of Medicine, Zagazig University, Sharkia, Egypt

Corresponding author: Ghada Ibrahim Ahmed, Mobile: +201202033360,

Email: Ghada.ibrahim1987@gmail.com

\begin{abstract}
Background: Acne vulgaris is an epidemic inflammatory disease of the human sebaceous follicle and represents the most common skin disease affecting about $85 \%$ of adolescents. Until recently, very little was known about the link between metabolic syndrome (MetS) and skin diseases. Over the past several years, it has been reported that MetS is potentially associated with numerous dermatological conditions, of which acne vulgaris.

Objective: To determine the relation between acne vulgaris and metabolic syndrome in adolescent males and females with different severities of acne vulgaris.

Material and Methods: A cross-sectional study on acne vulgaris patients, carried out in the outpatient clinic of Dermatology, Venereology and Andrology Department, Faculty of Medicine of Zagazig University Hospitals in the period from October 2017 to August 2018. This study included 60 patients of acne vulgaris of both sexes aged 10-19 years old.

Results: We found no statistically significant difference in age and sex between different patients with different disease severity. Also, no significant difference in fasting blood glucose, waist circumference, triglycerides, HDL, and blood pressure between different patients with different disease severity. There was a statistically significant difference in waist circumference and diastolic blood pressure only. There was no significant correlation between disease severity and metabolic syndrome criteria in the studied group.

Conclusion: Face is the commonest site affected. No association was found between acne vulgaris and metabolic syndrome in our study. No significant difference between different patients with different disease severity and (laboratory data, blood pressure).
\end{abstract}

Keywords: Acne vulgaris, Metabolic syndrome, adolescents.

\section{INTRODUCTION}

Acne vulgaris affects approximately $85 \%$ of the adolescent population. It affects over $90 \%$ of males and $80 \%$ of females in all ethnic groups ${ }^{(\mathbf{1})}$. In Egypt, El-hamd et al. ${ }^{(2)}$ reported the prevalence of acne in $60 \%$ of female secondary school students.

Acne vulgaris is a sebaceous gland disease related to inflammation and metabolism. The sebum secretion of sebaceous glands is mainly influenced by hormones, especially dihydrotestosterone. Although the composition of sebum is roughly the same in people with or without acne, acne populations are always accompanied by varying degrees of excessive secretion and seborrhea. ${ }^{(3)}$

Experimental evidence has indicated that the mechanistic target of rapamycin (mTOR) expression levels in the skin lesions of acne patients is positively correlated with the magnitude of insulin resistance. The activity of the mechanistic target of rapamycin complex 1 (mTORC1) was found to be elevated in the glands and injured skin of patients with acne compared with those who had no acne. Enhanced mTORC1 signaling is a known characteristic of insulin resistance, obesity, and $\mathrm{T}_{2} \mathrm{DM}^{(4)}$. Metformin, a commonly used drug for diabetes treatment that was identified as an mTORC1 inhibitor, is currently being considered as a candidate for treating insulin-resistant skin diseases, including acne vulgaris. A reduction of acne was observed in young males who adopted a hypoglycemic diet in conjunction with metformin therapy ${ }^{(5)}$.

Overall, young patients with acne have a higher tendency to develop insulin resistance, which may be a stage of prediabetes, and these patients may eventually go on to develop T2DM. Thus, close observation of insulinresistant acne patients is clinically significant for controlling disease progression ${ }^{\left({ }^{6}\right.}$.

The present study was aimed to determine the relation between acne vulgaris and metabolic syndrome in adolescent males and females with different severities of acne vulgaris. 


\section{MATERIALS AND METHODS}

\section{Ethical approval: This study is approved by the IRB} of Zagazig university at no. \#: 3830-9-7-2017. All patients gave their informed consent before the study.

\section{Technical design:-}

Setting: The study was carried out in the Dermatology, Venereology, and Andrology Department Faculty of Medicine, Zagazig University Hospitals.

Type of study: Cross-sectional study

Patients: A total number of patients of acne vulgaris (5 males and 55 females) were included in the study aged from 10-19 years old. They were selected from the Outpatient clinic of Dermatology, Venereology, and Andrology at Zagazig University Hospitals. In the period from October 2017 to August 2018 after obtaining the approval of the research ethics committee of Zagazig University Hospital.

Inclusion criteria: Age: 10-19 years. Sex: Both sexes. Acne vulgaris adolescents who are not using the treatment for their acne at least for 6 months.

Exclusion criteria: Patients who had used any treatment for acne within the last 6 months.

\section{Operational design:}

Steps of study: each patient was subjected to:

I-Complete history taking: A history including age, gender, the onset of acne, diabetes mellitus, hypertension, and concomitant medications taken.

\section{Complete general examination: General} examination of body systems was performed to discover the associated medical condition. General physical examination including the recording of blood pressure and waist circumference were done. Blood sampling: 3 $\mathrm{ml}$ whole blood were drawn from all the subjects after an overnight fast under complete aseptic condition and were divided into three portions: $1 \mathrm{~mL}$ of whole blood was collected into fluoride-containing tubes for estimation of fasting blood glucose. $2 \mathrm{~mL}$ was collected into plain tubes for 30-60 minutes for clotting then centrifuged at 3000 round per minute for 10 minutes; serum samples were separated into another set of tubes and kept frozen at $-20^{\circ} \mathrm{C}$ until the analysis for estimation of serum triglycerides and HDL cholesterol.

III-Local dermatological examination: Local dermatological examination of the site of acne vulgaris in the body. Local dermatological examination Results: including examination of the site of acne vulgaris in the body and measuring of acne vulgaris score was done by using the global acne grading system ${ }^{(7)}$.

\begin{tabular}{|c|c|}
\hline \multicolumn{2}{|c|}{ The global acne grading system. ${ }^{(7)}$. } \\
\hline Location & Factor \\
\hline Forehead & 2 \\
\hline Right cheek & 2 \\
\hline Left cheek & 2 \\
\hline Nose & 1 \\
\hline Chin & 1 \\
\hline Chest and upper back & 3 \\
\hline $\begin{array}{l}\text { Note: Each type of } \\
\text { depending on severity } \\
=1 \text {, papules }=2 \text {, pustu } \\
\text { score for each area } \\
\text { using the formula: Lo } \\
\text { (0-4). The global scor } \\
\text { and acne severity wa } \\
\text { score. A score of } 1-18 \\
\text { moderate; } 31-38 \text {, seve }\end{array}$ & $\begin{array}{l}\text { given a value } \\
=0 \text {, comedones } \\
\text { nodules }=4 \text {. The } \\
\text { e) is calculated } \\
\text { Factor } \times \text { Grade } \\
\text { of local scores, } \\
\text { sing the global } \\
\text { red mild; 19-30, } \\
\text { very severe }\end{array}$ \\
\hline
\end{tabular}

Research investigations: Determination of fasting blood glucose level. Determination of Lipid Profile (Serum triglycerides and HDL cholesterol) at the University Hospital laboratory.

\section{Statistical analysis}

All data were collected, tabulated, and statistically analyzed using SPSS 24.0 for windows (SPSS Inc., Chicago, IL, USA). Data were tested for normal distribution using the Shapiro Walk test. Qualitative data were represented as frequencies and relative percentages. Quantitative data were expressed as mean \pm SD (Standard deviation) for parametric and median and range for nonparametric data. Independent T-test and Mann Whitney tests were used to calculate the difference between quantitative variables in two groups for parametric and non-parametric variables respectively. One way ANOVA test was used to compare between more than two dependent groups. Pearson's correlation coefficient was used for correlating variables.

The (+) sign was considered as an indication for direct correlation and the (-) sign as an indication for inverse correlation, also we consider values near to 1 as strong correlation \& values near 0 as weak correlation. All statistical comparisons were two-tailed with a significance level of $\mathrm{P}$-value $\leq 0.05$ indicates significant, $\mathrm{p}<0.001$ indicates a highly significant difference while $P>0.05$ indicates a Non-significant difference. 
Table (1): Age and sex distribution of the studied group

\begin{tabular}{|c|c|c|}
\hline Variable & \multicolumn{2}{|c|}{$\begin{array}{c}\text { The studied group }(60) \\
\text { Mean } \pm \text { SD }\end{array}$} \\
\hline \multicolumn{3}{|c|}{ - } \\
\hline (years): & \multicolumn{2}{|c|}{$17.2 \pm 1.3$} \\
\hline Variable & NO(60) & $\%$ \\
\hline \multicolumn{3}{|l|}{ Sex } \\
\hline Male & 5 & $8.3 \%$ \\
\hline Female & 55 & $91.7 \%$ \\
\hline
\end{tabular}

This table shows that the age of the study group was (17.2 \pm 1.3$)$ ranged from (14-19) and (91.7\%) of them were females.

Table (2): Clinical finding data of the studied group

\begin{tabular}{|c|c|c|}
\hline Examination & \multicolumn{2}{|c|}{$\begin{array}{c}\text { The studied group }(60) \\
\text { Mean } \pm \text { SD }\end{array}$} \\
\hline - $\quad$ Systolic blood pressure $(\mathrm{mmHg})$ & \multicolumn{2}{|c|}{$112.6 \pm 9.1$} \\
\hline - Diastolic blood pressure $(\mathrm{mmHg})$ & \multicolumn{2}{|c|}{$72.9 \pm 6.9$} \\
\hline - Waist circumference $(\mathrm{cm})$ & \multicolumn{2}{|c|}{$82.1 \pm 7.3$} \\
\hline Clinical history & NO (60) & $\%$ \\
\hline \multicolumn{3}{|l|}{ The global acne grading system } \\
\hline $\begin{array}{ll}\text { - } & \text { Mild } \\
\text { - } & \text { Moderate } \\
\text { - } & \text { Severe }\end{array}$ & $\begin{array}{c}29 \\
22 \\
9\end{array}$ & $\begin{array}{l}48.3 \% \\
36.7 \% \\
15.0 \%\end{array}$ \\
\hline
\end{tabular}

This table shows that the systolic blood pressure of the study group ranged from (90-140), diastolic blood pressure ranged from (50-90), their waist circumference ranged from (70-112), (48.3\%) had mild disease severity and $(36.7 \%)$ were moderate.

Table (3): Sites of the lesion in the studied group

\begin{tabular}{|c|c|c|}
\hline Variable & NO.(60) & $\%$ \\
\hline - Face & 49 & $81.7 \%$ \\
\hline - Back & 1 & $1.7 \%$ \\
\hline - $\quad$ Chest & 1 & $1.7 \%$ \\
\hline - Face, back, and chest & 1 & $1.7 \%$ \\
\hline - $\quad$ Face and shoulders & 4 & $6.7 \%$ \\
\hline - Face, back and shoulders & 1 & $1.7 \%$ \\
\hline - Face and back & 1 & $1.7 \%$ \\
\hline - Back and shoulders & 2 & $3.3 \%$ \\
\hline
\end{tabular}

This table shows that face is the commonest site affected (81.7\%) followed by face and shoulders affection $(6.7 \%)$.

Table (4): Laboratory data of the studied group

\begin{tabular}{|c|c|}
\hline Variable & $\begin{array}{c}\text { The studied group }(60) \\
\text { Mean } \pm \text { SD }\end{array}$ \\
\hline - $\quad$ Fasting blood glucose $(\mathrm{mg} / \mathrm{dl})$ & $81.6 \pm 6.2$ \\
\hline - $\quad$ Triglycerides $(\mathrm{mg} / \mathrm{dl})$ & $96.3 \pm 17.4$ \\
\hline - $\operatorname{HDL~}(\mathrm{mg} / \mathrm{dl})$ & $37.1 \pm 5.5$ \\
\hline
\end{tabular}

This table shows that fasting blood glucose of the study group ranged from (69-97) $\mathrm{mg} / \mathrm{dl}$, triglycerides ranged from $(64-137 \mathrm{mg} / \mathrm{dl})$ and HDL ranged from $(28-60 \mathrm{mg} / \mathrm{dl})$.

Table (5): Prevalence of the metabolic syndrome criteria in the acne cases 


\begin{tabular}{|c|c|c|}
\hline \multicolumn{3}{|l|}{ Fasting blood glucose } \\
\hline - Normal & 60 & $100.0 \%$ \\
\hline - Abnormal & 0.0 & $0.00 \%$ \\
\hline \multicolumn{3}{|l|}{ Blood pressure } \\
\hline - Normal & 58 & $96.7 \%$ \\
\hline - Abnormal & 2 & $3.3 \%$ \\
\hline \multicolumn{3}{|l|}{ HDL } \\
\hline - $\quad$ Normal & 13 & $21.7 \%$ \\
\hline - Abnormal & 47 & $78.3 \%$ \\
\hline \multicolumn{3}{|l|}{ Triglycerides } \\
\hline - $\quad$ Normal & 60 & $100.0 \%$ \\
\hline - Abnormal & 0.0 & $0.00 \%$ \\
\hline \multicolumn{3}{|l|}{ Waist circumference } \\
\hline - Normal & 53 & $88.3 \%$ \\
\hline - Abnormal & 7 & $11.7 \%$ \\
\hline \multicolumn{3}{|l|}{ Metabolic syndrome } \\
\hline - $\quad$ Fulfilling criteria & 0.0 & $0.00 \%$ \\
\hline - Not Fulfilling criteria & 60 & $100.0 \%$ \\
\hline
\end{tabular}

This table shows that the percentage of abnormalities in fasting blood glucose, waist circumference, triglycerides, HDL, and blood pressure among the studied group (no one in the study group fulfilling the metabolic syndrome criteria).

Table (6): Comparing metabolic syndrome criteria between patients with different disease severity

\begin{tabular}{|c|c|c|c|c|c|}
\hline Variable & $\begin{array}{c}\text { Mild } \\
\text { Mean } \pm \text { SD (29) }\end{array}$ & $\begin{array}{c}\text { Moderate } \\
\text { Mean } \pm \text { SD (22) }\end{array}$ & $\begin{array}{c}\text { Severe } \\
\text { Mean } \pm \text { SD (9) }\end{array}$ & F-test & p-value \\
\hline - $\quad$ Systolic blood pressure $(\mathrm{mmHg})$ & $111.9 \pm 7.5$ & $111.7 \pm 11$ & $114.5 \pm 9.1$ & 0.4 & 0.6 \\
\hline - Diastolic blood pressure $(\mathrm{mmHg})$ & $72.6 \pm 7.3$ & $73.7 \pm 7.9$ & $74 \pm 5.3$ & 0.6 & 0.5 \\
\hline - Waist circumference $(\mathrm{cm})$ & $83.7 \pm 7.9$ & $79.8 \pm 5.1$ & $84.2 \pm 8.3$ & 1.7 & 0.2 \\
\hline - $\quad$ Fasting blood glucose $(\mathrm{mg} / \mathrm{dl})$ & $83.5 \pm 7.2$ & $79.8 \pm 4.5$ & $82.7 \pm 7.3$ & 1.8 & 0.2 \\
\hline - Triglycerides $(\mathrm{mg} / \mathrm{dl})$ & $96.9 \pm 17.2$ & $92.3 \pm 18.9$ & $99.4 \pm 20.5$ & 0.8 & 0.4 \\
\hline - $\operatorname{HDL}(\mathrm{mg} / \mathrm{dl})$ & $36.9 \pm 4.3$ & $36.4 \pm 4.4$ & $37.9 \pm 8.9$ & 1.4 & 0.2 \\
\hline
\end{tabular}

In this table, there was no statistically significant difference in fasting blood glucose, waist circumference, triglycerides, HDL, and blood pressure between different patients with different disease severity.

Table (7): Comparing metabolic syndrome criteria in the studied groups with different disease severity

\begin{tabular}{|c|c|c|c|c|c|c|c|c|}
\hline \multirow{2}{*}{ Variable } & \multicolumn{2}{|c|}{ Mild(29) } & \multicolumn{2}{|c|}{ Moderate(22) } & \multicolumn{2}{|c|}{ Severe(9) } & \multirow{2}{*}{$\chi^{2}$} & \multirow{2}{*}{ p-value } \\
\hline & No & $\%$ & No & $\%$ & No & $\%$ & & \\
\hline Blood pressure & & & & & & & & \\
\hline Normal & 28 & 96.6 & 21 & 95.5 & 9 & 100 & 0.4 & 0.8 \\
\hline Abnormal & 1 & 3.4 & 1 & 4.5 & 0.0 & 0 & & \\
\hline waist circumference & & & & & & & & \\
\hline Normal & 25 & 86.2 & 21 & 95.5 & 7 & 77.8 & 2.1 & 0.3 \\
\hline Abnormal & 4 & 13.8 & 1 & 4.5 & 2 & 22.2 & & \\
\hline HDL & & & & & & & & \\
\hline Normal & 7 & 24.1 & 3 & 13.6 & 3 & 33.3 & 1.7 & 0.4 \\
\hline Abnormal & 22 & 75.9 & 19 & 86.4 & 6 & 66.7 & & \\
\hline
\end{tabular}

In this table, there was no statistically significant difference in waist circumference, HDL, and blood pressure between patients with different disease severity.

Table (8): Correlation between Disease severity using the global acne grading system and patients characteristics in the studied group 


\begin{tabular}{|ll|c|}
\hline & $\mathbf{r}^{\wedge}$ & \multicolumn{1}{|c|}{$\mathbf{p}$} \\
\hline$\bullet \quad$ Age & -0.2 & $>0.05$ \\
\hline$\bullet \quad$ blood pressure (mmHg) & 0.02 & $>0.05$ \\
\hline$\bullet \quad$ Waist circumference (cm) & 0.01 & $>0.05$ \\
\hline$\bullet \quad$ Fasting blood glucose (mg/dl) & 0.2 & $>0.05$ \\
\hline$\bullet \quad$ Triglycerides (mg/dl) & 0.04 & $>0.05$ \\
\hline$\bullet \quad$ HDL(mg/dl) & -0.03 & $>0.05$ \\
\hline
\end{tabular}

${ }^{\wedge}$ pearson correlation

In this table, no significant correlation between disease severity and metabolic syndrome criteria in the studied group.

\section{DISCUSSION}

Acne is a widespread and complex skin diseaseaffecting individual of all ages, particularly between the ages of 15 to 17 years ${ }^{(8)}$. Although hyper seborrhea, follicular hyperkeratinization, Propionibacterium acnes colonization, and inflammation are found to be responsible in the pathogenesis, the mechanism of acne is not known clearly. The relationship between diet and acne has been uncertain in recent years ${ }^{(9)}$.

Some chronic diseases support the relationship between acne and insulin metabolism. Polycystic ovary syndrome (PCOS) is one of them, where obesity, hyperinsulinemia, insulin resistance, and hyperandrogenism are commonly observed. Acne is also a common occurrence in PCOS patients, as are high levels of IGF-1 and androgens. Both insulin and IGF induce the production of androgens while simultaneously inhibiting the hepatic synthesis of sex hormone-binding globulin (SHBG), therefore the bioavailability of androgens increases. The comedogenic effects of IGF-1 and high androgen levels are thought to be responsible for the acne seen in PCOS ${ }^{(10)}$.

The results of a previous study by Kaymak et al. ${ }^{(11)}$ suggest that the glycemic index of a diet and postprandial insulin levels do not play roles in the pathogenesis of acne, however, this study is the only one that contradicts other studies. Del Prete et al. ${ }^{(12)}$ provided evidence that young Italian males affected with acne had a high BMI and exhibited insulin resistance.

In our study, we clinically assessed acne patients according to Global Acne Grading System, there we found $48.3 \%$ had mild disease, $36.7 \%$ were moderate disease and $15 \%$ had severe disease. Mogaddam et al. ${ }^{(13)}$ documented comparable results, the severity of acne was mild in $64 \%$ of the patients, moderate in $32 \%$, and severe in $4 \%$.

We observed that the face is the commonest site affected among our patients, these observations were similar to Mogaddam et al. ${ }^{(13)}$ and Nagpal et al. ${ }^{(14)}$ studies.
Female acne is becoming more and more common ${ }^{(15)}$, previous literature stating that females were more vulnerable to acne than males in all age groups ${ }^{(\mathbf{1 6})}$.

In our study, we observed no statistically significant difference in age and sex between different patients with different disease severity, Mogaddam et al. ${ }^{(13)}$ and Nagpal et al. ${ }^{(14)}$ reported the same results.

We also found no statistically significant difference between different disease severity groups in waist circumference, SBP, and DBP. Inconsistency with our findings, Nagpal et al. ${ }^{(14)}$ found that the mean height, waist circumference, SBP, and DBP did not differ significantly among the different acne severity groups. However, Nagpal et al. ${ }^{(14)}$ observed that mean weight was found to be significantly different among the different severity groups. That the mean weight being significantly higher in the very severe acne group compared with that in the other severity groups, while BMI in the very severe acne group was significantly higher than that in the mild acne group.

We found also no significant difference among the different severity groups regarding fasting blood glucose, triglycerides, and HDL. In line with our finding Nagpal et al. ${ }^{(14)}$ documented no significant difference in fasting blood glucose and serum triglycerides and HDL.

We found a significant difference in waist circumference and diastolic blood pressure, but regarding systolic blood pressure, Fasting blood glucose Triglycerides, and HDL, there was no statistically significant difference between male and female patients. Inconsistent with our results, Beigh and Jain, ${ }^{(17)}$ found waist circumference was significantly different between male and female patients. However, they reported that gender differences were seen as significant statistically regarding HDL cholesterol and fasting glucose levels while they were similar regarding triglyceride and BP. This difference might be due to different cut-off points set as criteria for a metabolic syndrome-like WC and HDL-C.

In our study, we found no significant correlation between disease severity and metabolic syndrome criteria in the studied group. Also, Balta et al. ${ }^{(18)}$ did not find any 
association between BMI and severity of acne. However, Alan and Cenesizoglu, (19) observed a positive correlation between BMI and severity of acne.

Our result was in agreement with Balta et al. ${ }^{(18)}$ that showed no statistically significant difference in triglycerides (TG) in different disease severity in males or females. In this study, there was a statistically significant difference in waist circumference (an indicator of obesity) and diastolic blood pressure, but regarding systolic blood pressure and fasting blood glucose (FBG) there was no statistically significant difference between male and female patients.

On the opposite side in the study carried by Emiroğlu et al. ${ }^{(9)}$ included a group of 243 patients (male/female ratio 0.41) with severe acne and showed statistically significant differences with the control group in regards to BMI (as an indicator of obesity) and FBG, additionally statistically significant differences between cases and controls were found in the parameters FBG, systolic, and diastolic blood pressure.

In contrary to our study that showed no statistically significant difference in plasma lipid profile (TG, HDL) between different patients, Wen et al. ${ }^{(20)}$ study revealed that plasma lipid profile (TG, HDL) in acne patients does not just increase, but that there are also many acne patients in whom lipid profile is higher or lower than normal.

\section{CONCLUSION}

We found no association between acne vulgaris and metabolic syndrome. No significant difference between different patients with different disease severity and (laboratory data, blood pressure). Further clinical studies on a larger scale are needed to clarify the relation between acne vulgaris and metabolic syndrome criteria

\section{REFERENCES}

1. Alanazi M, Hammad S, Mohamed A (2018): Prevalence and psychological impact of Acne vulgaris among female secondary school students in Arar city, Saudi Arabia, in 2018. Electron Physician, 10(8):7224-7229.

2. El-Hamd M, Nada E, Moustafa M et al. (2017): Prevalence of acne vulgaris and its impact on the quality of life among secondary school-aged adolescents in Sohag Province, Upper Egypt. J Cosmet Dermatol., 16(3):370-3.

3. Tuchayi $S$, Makrantonaki E, Ganceviciene $R$ et al. (2015): Acne vulgaris. Nat Rev Dis Primers, 1:15029-33.

4. Melnik B (2018): Acne vulgaris: the metabolic syndrome of the pilosebaceous follicle. Clin Dermatol., 36(1):29-40.

5. Fabbrocini G, Izzo R, Faggiano A et al. (2016): Low glycaemic diet and metformin therapy: a new approach in male subjects with acne resistant to common treatments. Clin Exp Dermatol., 41(1):38-42.

6. Lian N, Chen M (2019): Metabolic Syndrome and Skin Disease; Potential Connection and Risk. Inter. J Dermatol and Venereol., 2(2): 89-93.

7. Adityan B, Kumari R, Thappa D (2009): Scoring systems in acne vulgaris. Indian J Dermatol Venereol Leprol., 75(3):323-6.

8. Law M, Chuh A, Lee A et al. (2010): Acne prevalence and beyond: acne disability and its predictive factors among Chinese late adolescents in Hong Kong. Clin Exp Dermaol., $35: 16-21$.

9. Emiroğlu N, Cengiz F, Kemeriz F (2015): Insulin resistance in severe acne vulgaris. Postepy Dermatol Alergol., 32(4):281-285.

10. Cordain L (2005): Implications for the role of diet in acne. Semin Cutan Med Surg., 24:84-91.

11. Kaymak Y, Adisen $\mathrm{E}$, Ilter $\mathbf{N}$ et al. (2007): Dietary glycemic index and glucose, insulin, insulin-like growth factor-1, insulin-like growth factor-binding protein 3, and leptin levels in patients with acne. J Am Acad Dermatol., 57:819-23.

12. Del Prete M, Mauriello M, Faggiano A et al. (2012): Insulin resistance and acne: a new risk factor for men? Endocrine, 42:555-60.

13. Mogaddam M, Ardabili N, Maleki $N$ et al. (2014): Correlation between the severity and type of acne lesions with serum zinc levels in patients with acne vulgaris. Biomed Res Int., 2014:474108.

14. Nagpal M, De D, Handa $S$ et al. (2016): Insulin Resistance and Metabolic Syndrome in Young Men With Acne. JAMA Dermatol., 152(4):399-404.

15. Preneau S, Dreno B (2012): Female acne - a different subtype of teenager acne? J Eur Acad Dermatol Venereol., 26(3):277-82.

16. Yang Y, Cheng Y, Lai C et al. (2007): Prevalence of childhood acne, ephelides, warts, atopic dermatitis, psoriasis, alopecia areata and keloid in Kaohsiung County, Taiwan: a community-based clinical survey. J Eur Acad Dermatol Venereol., 21(5):643-9.

17. Beigh S, Jain S (2012): Prevalence of metabolic syndrome and gender differences. Bioinformation., 8(13):613-616.

18. Balta I, Ekiz O, Ozuguz P et al. (2015): Insulin resistance in patients with post-adolescent acne. Int J Dermatol., 54(6):662-666.

19. Alan S, Cenesizoglu E (2014): Effects of hyperandrogenism and high body mass index on acne severity in women. Saudi Med J., 35(8):886-889.

20. Wen L, Jiang G, Zhang $X$ et al. (2015): Relationship between acne and psychological burden evaluated by ASLEC and HADS surveys in high school and college students from central China. Cell Biochem Biophys., 71(2):1083-8. 